\title{
Factors Affecting Trunk Fatigue and the Maximum Endurance Time of One-Leg Static Squat
}

\author{
Wenbao Li, Kai Way Li, Jingjing Chen, and Bin Feng
}

\begin{abstract}
One-leg static squat is a classic movement of lower limb muscle strength training and functional rehabilitation of the lower limbs. It is often used in the fields of exercise and exercise rehabilitation. This study was designed to determine the effects of gender, leg used, and back support mode on the maximum endurance time (MET) and CR-10 score of trunk fatigue under the two experimental conditions. Thirty adults participated in the study. A three-factor (sex, support legs, back support mode) experiment was performed. Descriptive statistics, analysis of variance (ANOVA), and correlation analysis were performed. The results showed that gender $(p<0.01)$, supporting leg $(p<0.05)$, and gender $\times$ back support mode $(p<0.01)$ were all significant on the MET. Gender $(p<0.01)$ was significant on the CR-10 score. There was a significant difference in the supporting time between the left and right legs of males and females. Whether it was supported by the wall or the yoga ball, the trunk of the males was significantly higher than those of the females. The coordination ability of the males' trunk and lower limbs was significantly different.
\end{abstract}

Index Terms-One-leg static squat, maximum endurance time; muscular fatigue, subjective rating.

\section{INTRODUCTION}

Muscle fatigue is a common phenomenon when people perform physical activities. It is defined as the ability to generate force or reduce the output power during exercise [1]. When the energy produced by the muscles is unable to maintain the existing work, muscles will fatigue until the maximum capacity for voluntary contraction is reached, resulting in ultimate exhaustion. The time from the start of physical exertion until exhaustion of muscles is called the maximum endurance time (MET). The MET may be used to evaluate the dynamic or static working ability of the muscles and may also be used to develop a training plan, muscle function rehabilitation program, and rest plan in industrial production [2].

The mechanism of muscle fatigue can be summarized as central fatigue theory, peripheral fatigue theory, nerve-muscle joint fatigue theory and comprehensive motor fatigue theory. Muscle fatigue can be divided into local body fatigue and

Manuscript received October 1, 2018; revised April 12, 2019.

Wenbao Li is with the Chung Hua University, Hsin-Chu, Taiwan, and a Faculty Member of Jilin Sport University, Changchun, China (e-mail: wenbaoli@126.com).

Kai Way Li is with the Department of Industrial Management, Chung Hua University, Hsin-Chu, Taiwan (e-mail: kai@ @hu.edu.tw)

Jingjing Chen is with College of Physics and Mechanical \& Electrical Engineering, Longyan University, Fujian, China (e-mail: 56339594@qq. com).

Bin Feng is Faculty of Tianjin College, University of Science \& Technology Beijing, China (e-mail: fbmailbox@126.com). systemic fatigue. Muscle fatigue can be measured by direct and indirect methods. The direct measurement method is to measure the degree of decline of the maximum voluntary contractile force of the muscle. The indirect test method measures the EMG of the muscle using an electromyography [3]. The MET of muscle completion and the subjective perception score of trunk fatigue can also be used as an indirect measure of muscle fatigue.

With the advancement of society and the sitting posture in the office is becoming more and more common, which makes people's lower limb strength generally decline. Even many fitness enthusiasts only pay attention to the upper limb strength training when performing resistance training, ignoring the strength training of the lower limbs, which leads to the imbalance of the upper and lower limb muscle ratios.

Leg static squat is the classic movement of lower limb muscle strength training. It can effectively strengthen the muscles of lower limbs, especially the strength of the quadriceps, not only improve the stability of the knee joint, but also improve the muscle's own control ability. Leg static squat against the wall is often used in athlete training and knee rehabilitation training [4]. In sports rehabilitation, one leg static squat is used as an evaluation method and training method for patellofemoral pain syndrome [5], [6]. If one leg is injured, muscle atrophy will occur. One leg static squat exercise has been used to strengthen the muscle strength of the affected thigh muscles, and to correct the difference in strength between the healthy side and the affected side and to avoid new damage can be caused by muscle imbalance. The angle of the knee joint is a key factor affecting the muscle strength of the trunk and lower limbs [7].

Strength training can not only improve bone density and delay bone loss but also increase muscle volume and circumference, and improve muscle working ability [8]. One leg static squat could increases the effects of training. The purpose of this study was to test the effects of leg usage, back support, and gender on the maximum endurance time (MET) in doing one leg static squat.

\section{METHODS}

\section{A. Subjects}

TABLE I: BASIC CHARACTERISTICS OF SUBJECTS (MEAN \pm SD)

\begin{tabular}{|c|c|c|c|c|c|}
\hline & Height $(\mathrm{cm})$ & Weight $(\mathrm{kg})$ & Age (year) & BMI & Fat $\%$ \\
\hline Male & $\begin{array}{c}182.27 \pm \\
6.54\end{array}$ & $64.46 \pm 7.07$ & $19.13 \pm 0.92$ & $19.39 \pm 1.57$ & $22.45 \pm 2.72$ \\
\hline Female & $\begin{array}{c}167.53 \pm \\
5.62\end{array}$ & $59.25 \pm 5.15$ & $18.73 \pm 0.70$ & $21.09 \pm 1.63$ & $31.82 \pm 3.62$ \\
\hline
\end{tabular}


Thirty college students, including 15 males and 15 females participated in this study. All subjects had no skeletal muscle damage within six months. All the subjects read and signed informed consent before joining the experiment. The basic characteristics of the subjects are shown in Table I.

\section{B. Materials and Apparatus}

One Swiss ball, one measuring tape, one weight scale, one measuring angle gauge, one stopwatch, one body composition analyzer (Korean inbody 5.5), and the Borg-10 scale were used.

\section{Experimental Design}

A 3-factor experiment was designed. The independent variables included gender (male, female), supporting legs (left and right legs), body support mode (back against the wall, back against the yoga ball). The dependent variable included the MET of one-leg static squat and subjective score on trunk fatigue using the Borg-10 scale. The MET refers to the time during which the subject strives to maintain one-legged rest until the muscles are fatigued and the subject could no longer maintain the stable posture.

\section{Experiment Procedure}

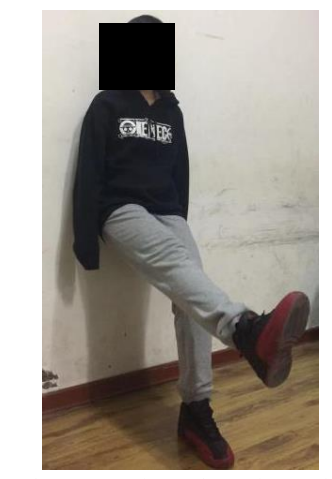

Fig. 1. Back against the wall.

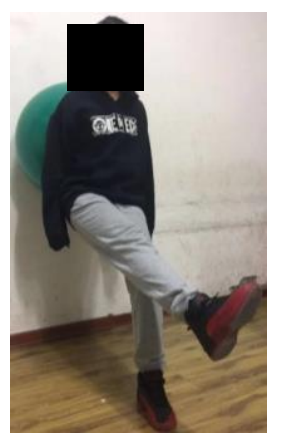

Fig. 2. Back supported by a yoga ball.

The subjects wore sneakers and sportswear to the lab. They took a 5 minute warm-up on the running platform, and then went through the tests. In the test, the upper body of the subject was straight up and the chest is upright, keeping the body upright, one foot supporting the ground with the knee joint at $120^{\circ}$ flexion (see Fig. 1). The toes were forward, the other foot was raised parallel to the ground, the back of the trunk was close to the wall, and the two arms were naturally drooping. Keeping this posture until the subject could no longer maintain. The research personnel immediately recorded the time of the test and the Borg-10 score of trunk fatigue. After the test, the subject took a break until he or she was fully recover from fatigue. A similar test was performed where the subject was supported by a yoga ball against the wall (See Fig. 2).

\section{RESULTS}

\section{A. Descriptive Statistical Analysis}

TABLE II: MAXIMUM ENDURANCE TIME TABLE (S)

Back against the wall Back to yoga ball

\begin{tabular}{llll}
\multicolumn{2}{c}{$($ mean \pm std $)$} & \multicolumn{2}{c}{$($ mean \pm std $)$} \\
\hline Left leg & Right leg & Left leg & Right leg
\end{tabular}

support support $\quad$ support $\quad$ support

Male $\quad 111.87 \pm 47.22 \quad 137.67 \pm 41.12 \quad 99.47 \pm 29.97 \quad 119.93 \pm 38.27$

Female $\quad 71.53 \pm 21.26 \quad 93.80 \pm 31.38 \quad 110.20 \pm 28.72 \quad 105.93 \pm 33.14$

Under the condition of backing against the wall, the MET of the left and right legs of the males were higher than those of the females. Under the condition of backing to the yoga ball, the METs of the left leg of the males were less than those of the females. The METs of the right legs of the males were higher than those of the females. For males, the back against wall had significantly higher MET than those of the yoga ball cases. For females, the yoga ball cases had significantly higher MET than those of the wall cases. The MET between the left and right legs for both males and females were significantly different.

Under the condition of wall support mode, when the left leg was supported, the CR-10 score for males and females were $6.13 \pm 1.92$ and $4.87 \pm 0.99$, respectively. The CR-10 score results are shown in Table III.

TABLE III: SUBJECTIVE RATING OF TRUNK FATIGUE

\begin{tabular}{ccccc}
\hline & \multicolumn{2}{c}{$\begin{array}{c}\text { Back against wall } \\
(\text { mean } \pm \text { std })\end{array}$} & \multicolumn{2}{c}{$\begin{array}{c}\text { Back to yoga ball } \\
(\text { mean } \pm \text { std })\end{array}$} \\
\cline { 2 - 5 } & $\begin{array}{c}\text { Left leg } \\
\text { support }\end{array}$ & $\begin{array}{c}\text { Right leg } \\
\text { support }\end{array}$ & $\begin{array}{c}\text { Left leg } \\
\text { support }\end{array}$ & $\begin{array}{c}\text { Right leg } \\
\text { support }\end{array}$ \\
\hline Male & $6.13 \pm 1.92$ & $6.27 \pm 1.87$ & $5.80 \pm 1.97$ & $5.87 \pm 2.00$ \\
Female & $4.87 \pm 0.99$ & $4.87 \pm 1.60$ & $5.53 \pm 1.51$ & $4.80 \pm 2.01$ \\
\hline
\end{tabular}

Under the condition of back against wall when the left and right legs were supported, there was no significant difference in the CR-10 between males and females. However, the CR-10 of males was significantly higher than those of the females. In the yoga ball cases when the left and right legs are supported, there was no significant difference in the CR-10 score. For females, the CR-10 of left leg conditions was higher than those of the right leg support cases. When the male was supported by the left and right legs of the wall cases, the CR-10 score was higher than that of the yoga ball cases. The CR-10 score was higher when the females were supported by left leg than the right leg only under the yoga ball condition.

\section{B. Analysis of Variance}

The ANOVA results indicated that the effects of gender $(p<0.01)$ and support legs $(p<0.05)$ were significant on the MET while the effects of the back support mode was not significant. The interaction between gender and back posture 
was significantly different $(p<0.01)$ (see Fig. 3). The interactions between gender and support legs, between the support leg and the back support mode, and the three way interactions for gender, support leg and the back support mode were all insignificant (See Table IV).

TABLE IV: AVONA TABLE FOR THE MET

\begin{tabular}{lrrrr}
\hline & SS & MS & $\mathrm{F}$ & Pr $>\mathrm{F}$ \\
\hline Sex** & 14344.53 & 14344.53 & 11.90 & 0.0008 \\
Leg* & 7744.13 & 7744.13 & 6.42 & 0.0126 \\
posture & 800.33 & 800.33 & 0.66 & 0.4168 \\
Sex*leg & 1498.13 & 1498.13 & 1.24 & 0.2673 \\
Sex*posture** & 12281.63 & 12281.63 & 10.19 & 0.0018 \\
Leg*posture & 1904.03 & 1904.03 & 1.58 & 0.2115 \\
Sex*leg*posture & 842.70 & 842.70 & 0.70 & 0.4049 \\
\hline$* P<0.05, * * P<0.01$ & & & &
\end{tabular}

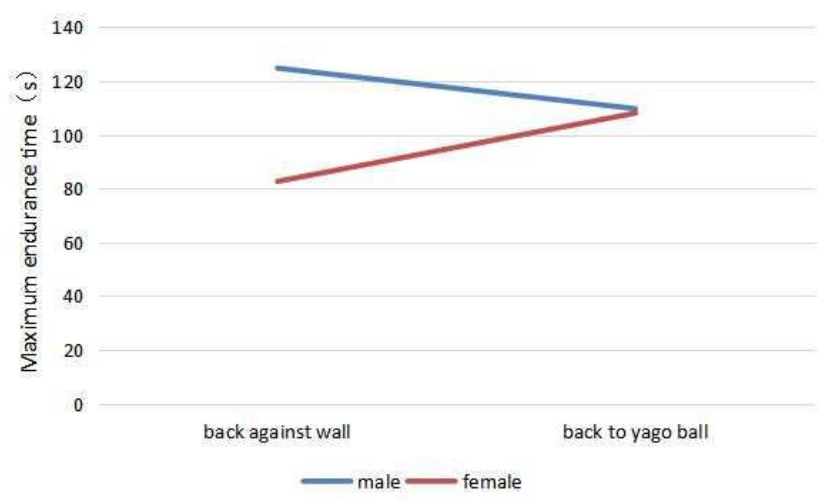

Fig. 3. The interaction between gender and back posture.

The gender and back support mode have an interaction effects on the MET. The MET of male backing against the wall was higher than those when backing on the yoga ball. For females, the MET when backing against the wall was lower than that when backing to the yoga ball. When the back was against the wall, the MET of the male was higher than those of the females. The MET for males and females when backing to the yoga ball was almost consistent.

TABLE V: ANOVA RESULTS FOR THE CR-10 SCORE

\begin{tabular}{lrrrr}
\hline & SS & MS & F & Pr $>$ F \\
\hline Sex** & 30.00 & 30.00 & 9.68 & 0.0024 \\
Leg & 2.00 & 2.00 & 0.62 & 0.4231 \\
posture & 0.82 & 0.82 & 0.26 & 0.6086 \\
Sex*leg & 0.29 & 0.29 & 0.09 & 0.7616 \\
Sex*posture & 1.31 & 1.31 & 0.42 & 0.5173 \\
Leg*posture & 2.02 & 2.02 & 0.65 & 0.4214 \\
Sex*leg*posture & 0.00 & $*$ & $*$ & $*$ \\
\hline$* P<0.05, * * P<0.01$ & & & &
\end{tabular}

For gender, support legs and back support mode, the ANOVA for the CR-10 score indicated that the gender was significant $(p<0.01)$. There was no significant difference between support leg and back support mode. There was no significant difference in the interaction between the three factors.

\section{DISCUSSION}

Static squat may be divided into two legs statics squat and one leg statics squat. It is also a classic movement to prevent and treat the "running knee". Through experiments, it was found that when the male and female completed backing against wall and the yoga ball with one leg statics squat, the MET of the left and right legs was significantly different. It was shown that under steady state, the MET of males was significantly higher than that of females, indicating that the strength and endurance of males' lower limb muscles are stronger than females. Therefore, in the steady state of statics training or lower limb knee function rehabilitation, we must first fully consider the gender differences, and then develop the program. Under the unsteady state of yoga ball, the difference in the MET between males and females was small. It may be because the coordination control ability of female is better than that of male. Male should consciously increase coordination ability training during normal exercise. Through experiments, it was found that the support time of the left and right legs of male and female was quite different. In the training, we should pay attention to the coordinated training of the left and right lower limb muscles to ensure the balanced development of the left and right muscle strength.

When the muscle strength of the left and right legs develops unbalanced, the neutral position of the pelvis will be affected. The pelvic rotation phenomenon will also occur. This could result in long-term weight bearing on one side of the leg and weakness of the contralateral thigh muscles such as the gluteal muscles and the hamstrings. Even muscle damage may eventually cause a lower cross syndrome, with one-leg static movement training, one can passively stretch the quadriceps of the support leg to make it equal length contraction. This not only strengthens the muscle strength, but also avoids muscle strain caused by improper dynamic movement. When the strength of the muscle is increased, the stability of the knee joint can be enhanced. Knee joint, as the most complicated joint in the human body, is more likely to be injured during normal life work and exercise. For example, the damage of the meniscus and the injury of the anterior cruciate ligament, it can be seen that the stability of the knee joint is strengthened to prevent the injury of the knee joint.

In the condition of backing to yoga ball, due to the intervention of the yoga ball, the difficulty of technical movement is increased, and the exerciser is required to perform stability training in an unstable state. We found that the MET of female in non-steady state was better than that under steady state, indicating that females' coordination, flexibility, stability of core muscle groups[8-9] and ability to control muscles were stronger than that of males. For the one-leg static movement, males should strengthen the training under the unstable state. Females should also increase the training under the steady state in order to improve the working ability of the lower limb muscles more effectively.

From the results of the CR-10 score, it was found that the degree of fatigue of males was significantly higher than that of females. There was a significant difference in the ability of coordination between the trunk and the lower limbs. The males' control ability was lower than that of the females, and the muscle differentiation ability was weak. Therefore, as a 
simple and easy exercise method, the single-legged static movement can not only evaluate the cooperative control ability of the trunk and the lower limb, but also serve as an effective method for training.

\section{CONCLUSION}

The MET was affected significantly by gender, and was also affected significantly by the interaction between gender and back support mode. Under steady state, the MET of males was significantly higher than that of females. In unstable state, females had higher left leg MET than those of males. On the other hand, the right leg METs of males were higher than those of females. The CR-10 score of trunk fatigue was affected by gender. Under steady state and unsteady state, the CR-10 of male was higher than that of female. The MET and subjective score of trunk fatigue were not correlated with body weight, BMI, body fat $\%$, thigh circumference and calf circumference. When using the one-leg static movement as a training method, it is necessary to fully consider the gender difference. The difficulty can be increased by changing the back support mode to increase the training effect.

\section{REFERENCES}

[1] J. D. Willson, M. L. Ireland, and I. Davis, "Core strength and lower extremity alignment during single leg squats," Medicine \& Science in Sports \& Exercise, vol. 38, no. 5, pp. 945-952, 2006.

[2] Z. Zhang, K. W. Li , W. Zhang et al., "Muscular fatigue and maximum endurance time assessment for male and female industrial workers," International Journal of Industrial Ergonomics, vol. 44, no. 2, pp. 292-297, 2014.

[3] K. W. Li, C. W. Wang, and R. Yu, "Modeling of predictive muscular strength for sustained one-handed carrying task," Work, vol. 52, no. 4, pp. 911-919, 2015

[4] R. Escamilla, N. Zheng, R. Imamura et al., "Cruciate ligament force during the wall squat and the one-leg squat," Medicine+ Science in Sports+ Exercise, vol. 41, no. 2, pp. 408, 2009.

[5] P. Levinger, W. Gilleard, and C. Coleman, "Femoral medial deviation angle during a one-leg squat test in individuals with patellofemoral pain syndrome," Physical Therapy in sport, vol. 8, no. 4, pp. 163-168, 2007.

[6] P. Levinger, W. L. Gilleard, and K. Sprogis, "Frontal plane motion of the rearfoot during a one-leg squat in individuals with patellofemoral pain syndrome," Journal of the American Podiatric Medical Association, vol. 96, no. 2, pp. 96-101, May 2006.

[7] J. E. Layne, and M. E. Nelson, "The effects of progressive resistance training on bone density: a review," Medicine and Science in Sports and Exercise, vol. 31, no. 1, pp. 25-30, 1999

[8] T. M. Handzel, "Core training for improved performance," JNSCA's Performance Training Journal, vol. 2, no. 6, pp. 26-30, 2003.

[9] W. B. Kibler, J. Press, and A. Sciascia, "The role of core stability in athletic function," Sports Medicine, vol. 36, no. 3, pp. 189-198, 2006

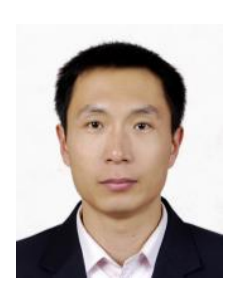

Wenbao Li was born in Changchun city, Jilin Province. He is studying in the Ph.D. program in engineering science, Chung Hua University, Hsin-Chu, Taiwan. He is also one of the faculty members with Jilin Sports University, China. His major field is in human engineering and human movement science.

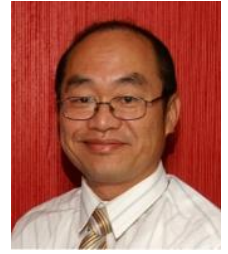

Kai Way Li is a professor with the Department of Industrial Management, Chung Hua University, Hsin-Chu, Taiwan. He received his Ph.D. degree from the Department of Industrial Engineering, Texas Tech University, USA. He has been conducting research in ergonomics including slip \& fall, manual materials handling, \& muscular fatigue.

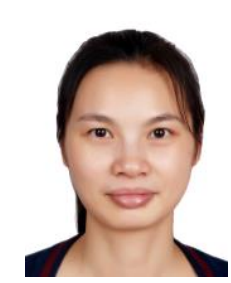

Jingjing Chen was born in Longyan city, Fujian Province, China. She received the M.S. degree in circuits and systems from Central China Normal University. She is currently pursuing the Ph.D. degree with College of Engineering, Chung Hua University, Hsin-Chu, Taiwan. She is also one of the faculty members with Longyan University.

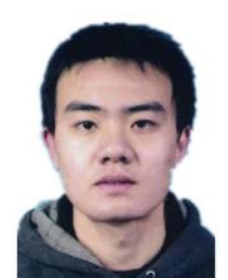

Bin Feng was born in Baoding city, Hebei Province, China. He is work in Tianjin College, University of Science and Technology Beijing, Tianjin, China. His major field is physical education and sport training. 